\title{
Resultados del manejo quirúrgico en meningioma esfeno-orbitario
}

\section{Results of the surgical management of an esphenoorbitary meningioma}

\author{
José Antoni Soriano Sánchez, ${ }^{*}$ Manuel Eduardo Soto García, ${ }^{*}$ \\ Saúl Solorio Pineda, ${ }^{\ddagger}$ José Alberto Israel Romero Rangel ${ }^{*}$
}

Citar como: Soriano SJA, Soto GME, Solorio PS, Romero RJAI. Resultados del manejo quirúrgico en meningioma esfeno-orbitario. An Med (Mex). 2021; 66 (1): 63-66. https://dx.doi.org/10.35366/99492

\section{RESUMEN}

Los meningiomas esfeno-orbitarios constan de dos componentes: hiperostosis esfenoidal extensa y crecimiento intradural. La invasión dural a menudo se extiende hasta el ala esfenoidal basal, fisura orbitaria superior, canal óptico seno cavernoso, fosa infratemporal y estructuras periorbitarias. El presente trabajo tiene como objeto demostrar el manejo quirúrgico de estos pacientes con las ventajas de la neuronavegación y la reconstrucción 3D de la placa craneal con esterolitografía. Mujer de 46 años de edad con disminución progresiva de la agudeza visual de ojo derecho de un año de evolución, posteriormente se agrega disminución de la movilidad ocular y deformidad en el mismo ojo. En la resonancia magnética simple y contrastada de encéfalo se observa tumoración extraaxial dependiente del ala menor del esfenoides del lado derecho con extensión en placa a techo orbitario y fosa media, con componente tumoral intraorbitario extraconal que desplaza estructuras intraoculares, compatible con meningioma esfeno-orbitario derecho. Se realiza craniectomía frontotemporal con osteotomía órbito-cigomática derecha, descompresión de hendidura esfenoidal y agujero óptico, guiada por neuronavegación (Simpson I), ulteriormente se realizó reconstrucción de defecto craneal con placa preformada en

\section{ABSTRACT}

Spheno-orbital meningiomas consist of two components: extensive sphenoid hyperostosis and intradural growth. The dural invasion often extends to the basal sphenoid wing, superior orbital fissure, cavernous sinus optic canal, infratemporal fossa and periotbitary structures. The purpose of this work is to demonstrate the surgical management of these patients, with the advantages of neuronavigation and $3 D$ reconstruction of the cranial plate with sterolithography. A 46-year-old woman with a progressive decrease in visual acuity of the right eye after one year of evolution, subsequently showed a decrease in ocular mobility and deformity of the right eye. Simple and contrasted magnetic resonance of the brain showed extra-axial tumor dependent on the minor wing of the sphenoid of the right side with an extension in the plate to the orbital roof and the middle fossa, with an extra-conal intraorbital tumor component displacing intraocular structures, compatible with right spheno-orbital meningioma. Frontotemporal craniectomy was performed with right orbito-zygomatic osteotomy and sphenoid cleft decompression and optic hole, guided by neuronavigation (Simpson I), subsequently performing cranial defect reconstruction with $3 D$ preformed methylmethacrylate plate. Spheno-orbital meningioma is a rare tumor of the base of the skull with intraosseous growth and hyperostosis

\footnotetext{
* Neurocirujano. Centro Médico ABC.

‡ Neurocirujano Centro Médico «Lic. Adolfo López Mateos», ISEM, Toluca, Estado de México.
}

Recibido: 10/06/2020. Aceptado: 03/03/2021.

\section{Correspondencia:}

Dr. José Alberto Israel Romero Rangel

Av. Carlos Graef Fernández Núm. 154, Col. Tlaxala Santa Fe, 05300, Cuajimalpa, México. Tel: 55 8201-5788.

E-mail: gmaisrael@gmail.com israel@neurosurgery.clinic 
3D de metilmetacrilato. El meningioma esfeno-orbitario es un tumor raro de la base del cráneo con crecimiento intraóseo e hiperostosis que clínicamente en la mayoría de los casos se manifiesta con proptosis, discapacidad visual. Es de importancia la resección tumoral agresiva con el fin de evitar recidiva tumoral y mejorar el pronóstico a largo plazo.

Palabras clave: Meningioma esfenoidal, esfeno-orbitario, craneoplastia, neuronavegación, estereolitografía.

\section{INTRODUCCIÓN}

Los meningiomas esfeno-orbitarios son lesiones infiltrantes de lento crecimiento. Se definen como meningiomas de la cresta esfenoidal con extensión intraorbitaria e hiperostosis del hueso temporal, se encuentran dentro de 2.5-9\% de meningiomas intracraneales. ${ }^{1-4}$ Estos tumores constan de dos componentes: hiperostosis esfenoidal extensa secundaria al crecimiento tumoral intraóseo y crecimiento intradural. ${ }^{1}$

La hiperostosis tiende a extenderse al ala esfenoidal, techo orbital, fisura orbital superior, canal óptico y proceso clinoides anterior. La invasión dural a menudo se extiende hasta el ala esfenoi- that clinically in most cases manifests with proptosis, visual impairment. Aggressive tumor resection is important in order to avoid tumor recurrence and improve long-term prognosis.

Keywords: Sphenoid meningioma, spheno-orbital, cranioplasty, neuronavigation, stereolithography.

dal basal, convexidad temporal, periórbita, fisura orbitaria superior, canal óptico y seno cavernoso. Otras estructuras que invade son la fosa infratemporal, el músculo temporal y estructuras de la periórbita como grasa periorbitaria y músculos extraoculares. ${ }^{3}$

El síndrome clínico característico de los meningiomas esfeno-orbitarios es el resultado de cambios patológicos óseos, intradurales e intraorbitarios; incluye la proptosis como la manifestación de presentación más común, seguida de compromiso visual y paresia ocular. $^{5}$

En estos tumores debe eliminarse al máximo, de forma segura, el hueso invadido y una abertura amplia del canal óptico de manera rutinaria en pacien-
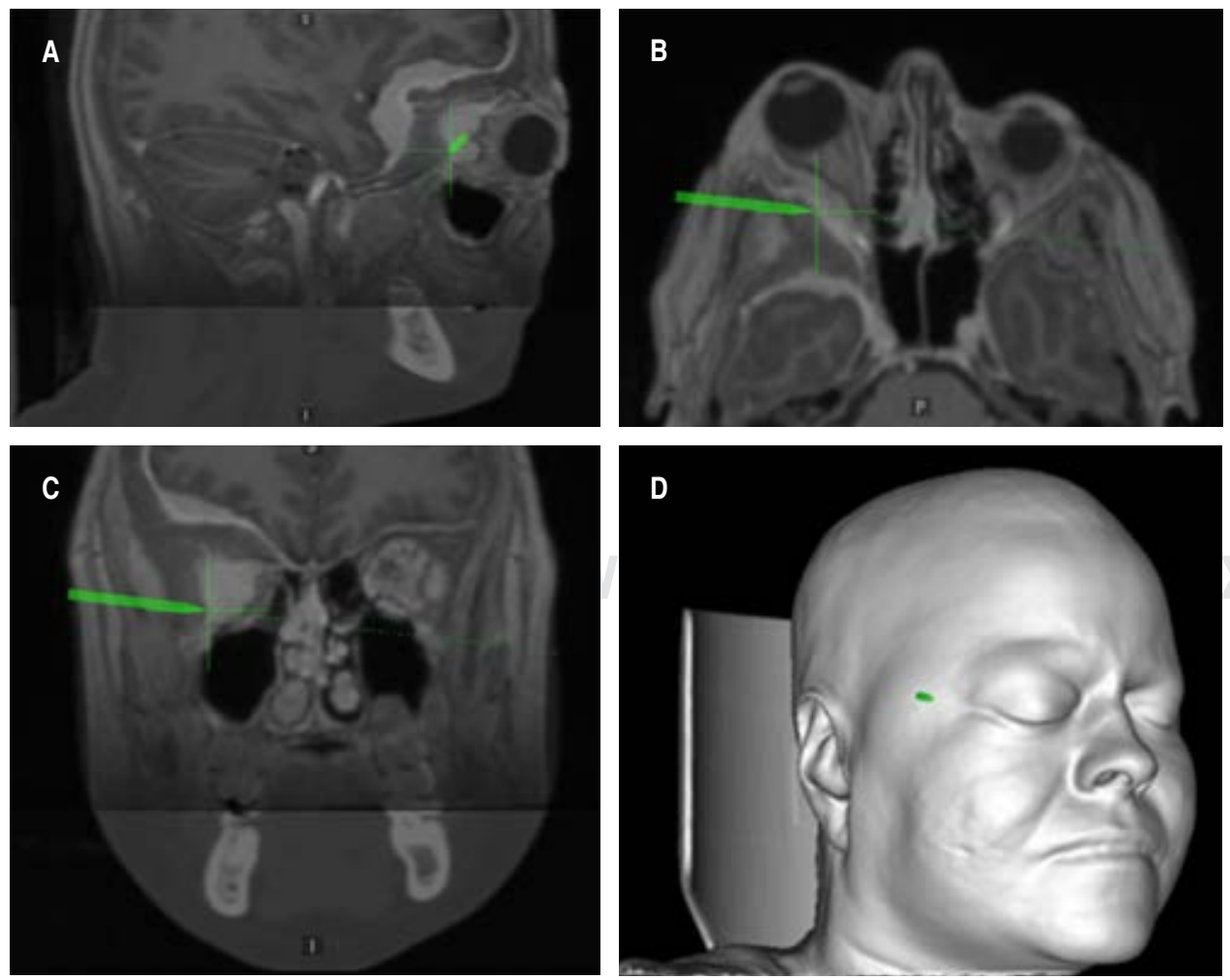

Figura 1:

A-C) Imagen de neuronavegador en sus tres planos (corona, axial y sagital), se observa tumoración extraaxial dependiente del ala menor del esfenoides del lado derecho con extensión del techo orbitario hacia la fosa craneal media y componente tumoral intraorbitario. D) Reconstrucción volumétrica en 3D, se observa marcada proptosis de ojo derecho. 

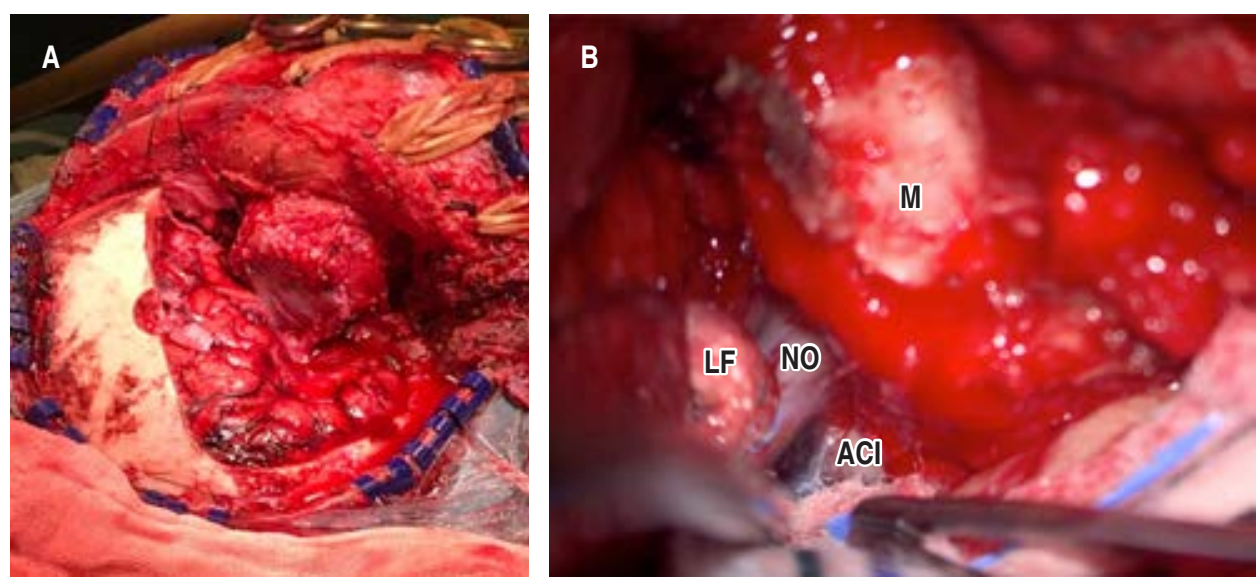

Figura 2:
A) Se observa tumoración dependiente del ala mayor del esfenoides con invasión hacia estructuras óseas vecinas. B) Relación de meningioma (M), esfeno-orbitario con nervio óptico (NO), arteria carótida interna (ACI) y lóbulo frontal (LF).

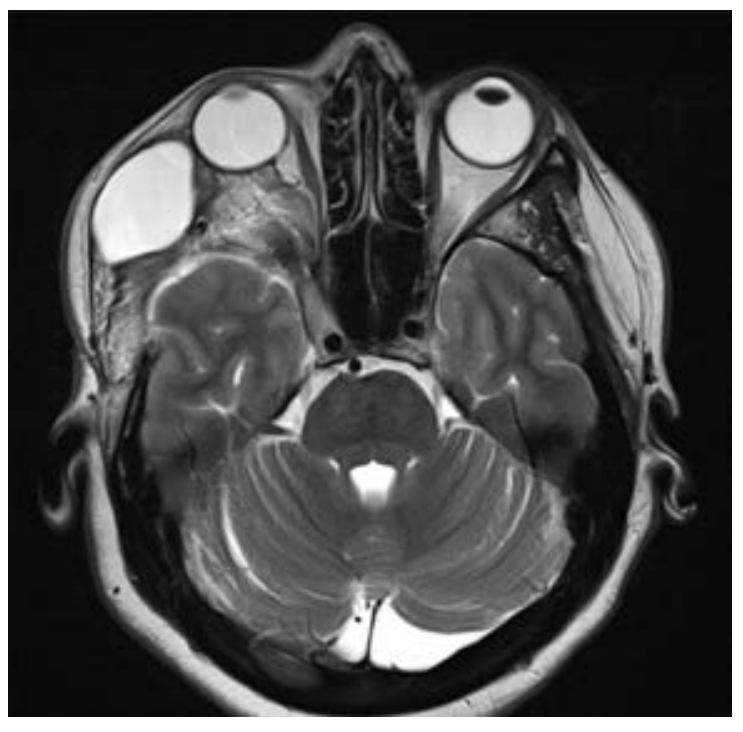

Figura 3: Resonancia magnética postoperatoria en secuencia T2 axial que muestra resección Simpson I de meningioma y fístula contenida.

tes con tumores de los ápices orbitarios u orbitarios difusos con una técnica microquirúrgica crítica a través de un abordaje supraorbitario-pterional. Ésta es la técnica de elección con el objetivo de mayor preservación funcional siendo siempre indispensable el seguimiento a largo plazo para evitar la recurrencia temprana, en caso de presentarse esta última considerar cirugía adicional e incluso radiocirugía para casos recurrentes o malignos. ${ }^{2,3,5}$

\section{CASO CLÍNICO}

Mujer de 46 años de edad presenta disminución progresiva de la agudeza visual de ojo derecho de un año de evolución, posteriormente se agrega disminución de la movilidad ocular y deformidad del mismo ojo. A la exploración neurológica con proptosis y disminución de la agudeza visual de ojo derecho, solamente detecta bultos, no distingue colores, con paresia de músculos extraoculares de predominio recto superior con supraversión limitada de ojo derecho, reflejos fotomotor y consensual disminuidos del lado derecho, sin compromiso de la fuerza y sensibilidad.

En la resonancia magnética simple y contrastada de encéfalo se observa tumoración extraaxial dependiente del ala menor del esfenoides del lado derecho con extensión en placa a techo orbitario y fosa media, componente tumoral intraorbitario extraconal que desplaza estructuras intraoculares, compatible con meningioma esfeno-orbitario derecho (Figura 1).

Se realiza incisión pterional ampliada, craniectomía frontotemporal con osteotomía órbito-cigomática derecha, descompresión de hendidura esfenoidal y agujero óptico, guiada por neuronavegación, resección de implante dural con componente tumoral óseo frontoorbitario (Simpson I) y descompresión orbitaria extraconal con plastia dural (Figuras 2 y 3 ).

Se realiza procedimiento quirúrgico ya comentado sin presentar déficit neurológico agregado, se realiza estudio histopatológico con H\&E confirmando diagnóstico de meningioma transicional con componente microquístico (OMS: grado I) así como estudio confirmatorio de inmunohistoquímica (receptores de progesterona positivos, antígeno de membrana antiepitelial positivo, vimentina positivo, Ki-67 positivo al 3\%).

Posterior a cirugía se realiza resonancia magnética simple y contrastada de control sin evidencia de tumor residual, por lo que no ameritó radioterapia adyuvante, manteniéndose en vigilancia estrecha y libre de actividad tumoral a dos años de seguimiento y se lleva a cabo 
An Med (Mex) 2021; 66 (1): 63-66

reconstrucción de defecto craneal con placa preformada en 3D de metilmetacrilato (Figura 4).

\section{DISCUSIÓN}

Los meningiomas esfeno-orbitarios son tumores infrecuentes a nivel de la base del cráneo, tienen una infiltración tumoral significativa e hiperostosis que involucra predominantemente el ala menor del hueso esfenoides que puede extenderse al ala esfenoidal mayor, la clinoides anterior y los huesos frontal y temporal, presentándose con proptosis insidiosa e indolora y/o compromiso visual ${ }^{6}$ como es el caso de nuestra paciente.

Essa y colaboradores escribieron una serie de 15 casos de meningioma esfenoidal en placa, donde el síntoma de presentación más frecuente fue la proptosis. Estos autores reportaron tasas de resección completa y mejoría en la proptosis de $67 \%$ cada una, ${ }^{7}$ concluyendo que este tipo de meningiomas conlleva una elevada morbilidad y complejidad para su resección, que requiere técnicas microquirúrgi-
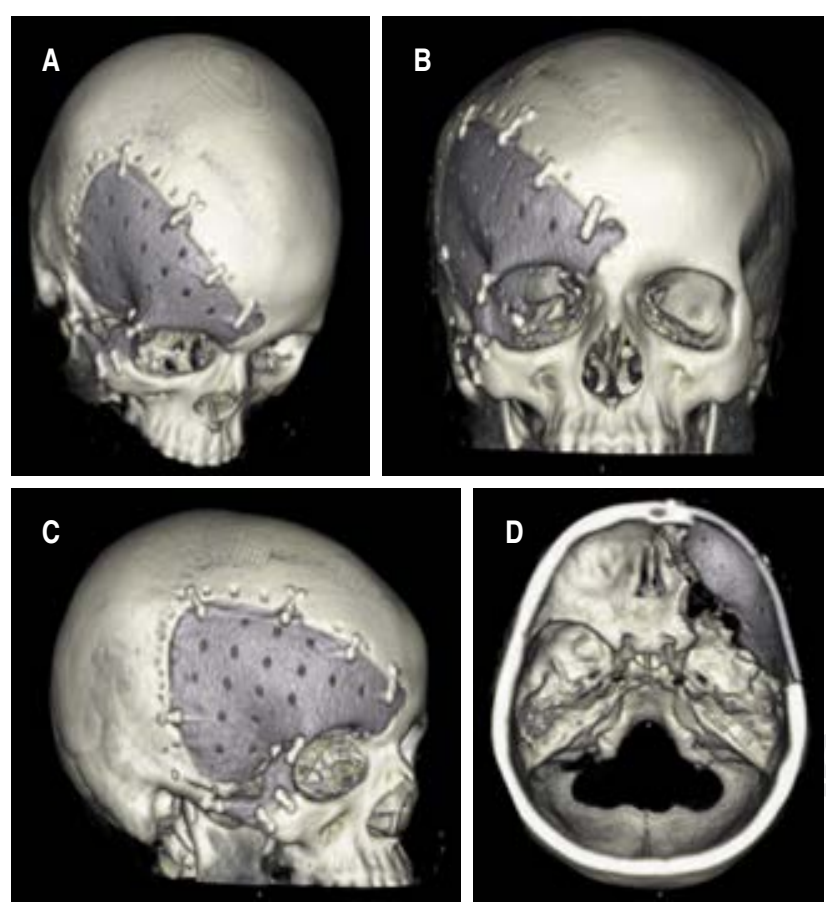

Figura 4: Control tomográfico de reconstrucción craneal con craneoplastia por medio de estereolitografía computarizada 3D. A) Proyección anterolateral. B) Frontal. C) Lateral. D) Corte axial en 3D que muestra fosas craneales. cas avanzadas y una reconstrucción meticulosa para lograr apropiados resultados funcionales y estéticos. En el presente caso obtuvimos una resección completa y también recuperación de la proptosis. Dentro del espectro de técnicas quirúrgicas, Buttrick y colaboradores consideran que la clinoidectomía etapificada (destechamiento orbitario, destechamiento del canal orbitario, remoción del puntal orbitario y clinoidectomía anterior) como un paso clave en la resección de los meningiomas esfenoidales; sin embargo, esta consideración cobra especial relevancia cuando existe compromiso del nervio óptico, ${ }^{8}$ situación que no ocurrió en nuestra paciente, por lo que preferimos realizar un abordaje orbitocigomático con destechamiento orbitario, dada la localización más lateral de la lesión.

\section{CONCLUSIONES}

El meningioma esfeno-orbitario es un tumor raro de la base del cráneo con crecimiento intraóseo e hiperostosis que clínicamente en la mayoría de los casos se manifiesta con proptosis, discapacidad visual. Es de importancia la resección tumoral agresiva con el fin de evitar recidiva tumoral y mejorar el pronóstico a largo plazo.

\section{REFERENCIAS}

1. Gonen L, Nov E, Shimony N, Shofty B, Margalit N. Sphenoorbital meningioma: surgical series and design of an intraoperative management algorithm. Neurosurg Rev. 2018; 41 (1): 291-301.

2. Mariniello G, Bonavolonta G, Tranfa F, Maiuri F. Management of the optic canal invasion and visual outcome in spheno-orbital meningiomas. Clin Neurol Neurosurg. 2013; 115 (9): 1615-1620.

3. Nagahama A, Goto T, Nagm A, Tanoue Y, Watanabe Y, Arima $\mathrm{H}$ et al. Spheno-orbital meningioma: surgical outcomes and management of recurrence. World Neurosurgery. 2019; 126: e679-687. Available from: https://doi.org/10.1016/j. wneu.2019.02.123.

4. Castellano F, Guidetti B, Olivecrona H. Pterional meningiomas en plaque. J Neurosurg. 1952; 9 (2): 188-196.

5. Shrivastava RK, Sen C, Costantino PD, Della Rocca R. Sphenoorbital meningiomas: Surgical limitations and lessons learned in their long-term management. J Neurosurg. 2005; 103 (3): 491-497.

6. Bowers CA, Sorour M, Patel BC, Couldwell WT. Outcomes after surgical treatment of meningioma-associated proptosis. J Neurosurg. 2016; 125 (3): 544-550.

7. Essa AA, Hamdan AR. Sphenoid meningioma enplaque with proptosis: Surgical excision, reconstruction and outcome. Clin Neurol Neurosurg. 2018; 167: 147-156. Available from: https:// doi.org/10.1016/j.clineuro.2018.02.028.

8. Buttrick S, Morcos JJ, Elhammady MS, Wang AC. Extradural clinoidectomy for resection of clinoidal meningioma. Neurosurg Focus. 2017; 43 (videosuppl2): V10. Available from: https://thejns.org/view/journals/neurosurg-focus/43/ videosuppl2/2017.10.FocusVid.17363.xml. 